\title{
Effect of the Beam Parameters on the Non-linear Performance in Broadband Helix TWTs
}

\author{
R. O. Jenkins and R. G. Carter \\ Engineering Department, Lancaster University, Lancaster LA1 4YR \\ email: r.o.jenkins@lancaster.ac.uk, r.carter@lancaster.ac.uk
}

Tel: 441542 593326, Fax: 441524381707

Today's communications systems demand helix TWTs which are highly efficient and linear across a specified bandwidth. These three requirements rely upon ensuring the phase of the slow-wave structure and the electron beam are properly matched throughout the tube.

The helix slow-wave structure can be designed to optimise the bunching of the electron beam and to synchronise the forward wave on the helix with the decreasing beam phase velocity as the electrons transfer their energy to the RF circuit ${ }^{1}$. It has been shown that a design can be achieved by this means which meets the necessary systems requirements at the centre frequency ${ }^{1}$. By adjusting the properties of the electron beam however, the nonlinear and the broadband performance of the tube can also be altered as a result of the changed phase velocity of the slow-space charge wave.

Research has been carried out which investigated the effect of the beam parameters on the nonlinear performance of a helix TWT across a specified broad frequency band. The beam parameters include the beam voltage (at constant DC power) and the beam radius. A large signal (1D and 2.5D) computer program ${ }^{2}$ was used for this purpose, which models the interaction processes in a helix TWT and generates transfer characteristics for a given design at specified frequency values. An intermodulation analysis software (IMAL 2-0) ${ }^{3}$ was also used to compute the nonlinear performance for multi-carrier signals.

Fig. 1 shows how the beam voltage (from 4.76 to $5.24 \mathrm{KV}$ ) influences the AM/AM transfer characteristics across a specified broad bandwidth $(10.7$ to $12.75 \mathrm{GHz})$. Figures 2 and 3 show the corresponding efficiency and gain characteristics respectively. The results show that consistency in the performance across the bandwidth can change considerably for different drive levels. Fig. 4 shows the output backoff that is required for an acceptable carrier-to-intermodulation noise ratio of around $-21 \mathrm{dBc}$, which is usually greater than $4 \mathrm{~dB}^{4}$. The design of the TWT for consistent performance across the bandwidth should therefore be targeted at drive levels well below that of saturation. The results also reveal that for a given pitch profile optimised for, say, efficiency, varying the beam voltage can effectively improve either the linearity or the broadband performance of the design, at the expense of efficiency.

1. R. O. Jenkins and R. G. Carter, "Design of the pitch profile for high linearity helix TWT Amplifiers", Proc. Conf. Displays and Vacuum Electronics, GarmischPartenkirchen, Germany, May 2001. 
2. V. Strivastava and S. N. Joshi, "Improved nonlinear model for multi-signal analysis of helix TWTs", Proc. Inst. Elec. Eng. H, vol. 139, no. 2, pp. 129-134, 1992.

3. R. G. Carter, W. Bosch and V. Strivastava, "Computer simulation of intermodulation distortion in broad-band, multi-carrier travelling-wave tube amplifiers", Proc. Conf. Displays and Vacuum Electronics, GarmischPartenkirchen, Germany, April 1998.

4. R. O. Jenkins and R. G. Carter, "Optimisation of the transfer curves of multicarrier power amplifiers for low intermodulation distortion", in Proc. EPSRCPREP 2001, 9-11, April 2001.

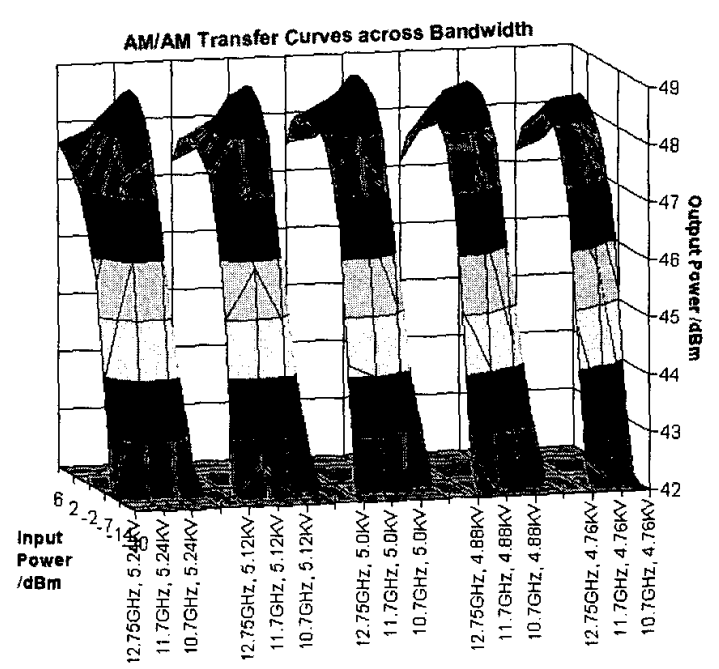

Fig 1: AM/AM transfer characteristics, for different beam voltages, across the bandwidth.

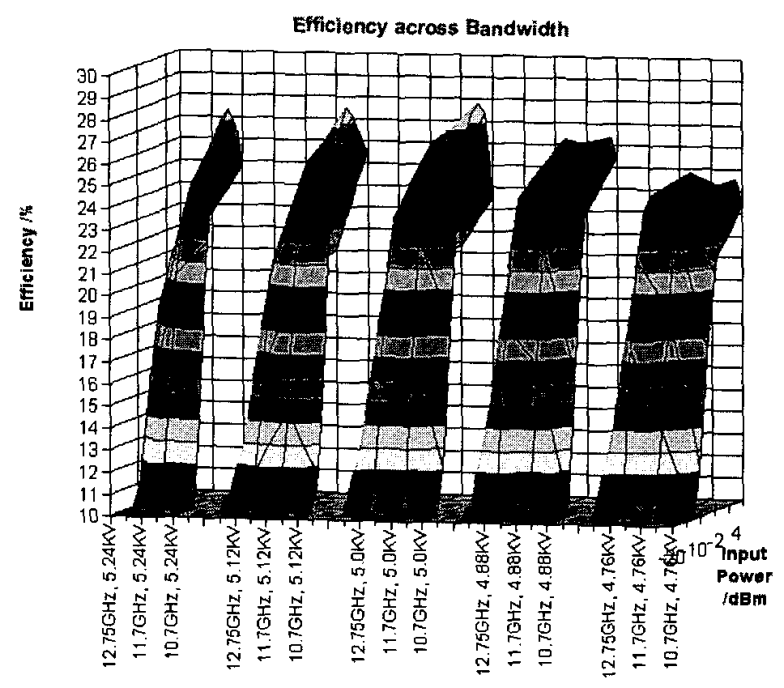

Fig 2: Efficiency versus input power, for different beam voltages, across the bandwidth.

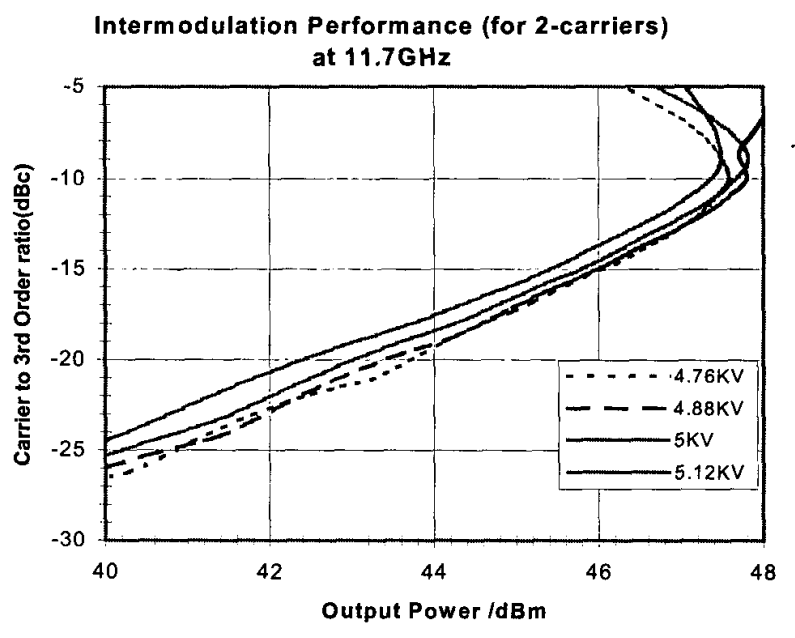

Fig 4: Carrier to third-order-power as a function of output power for different beam voltages.
Fig 3: Gain versus input power, for different beam voltages across the bandwidth.

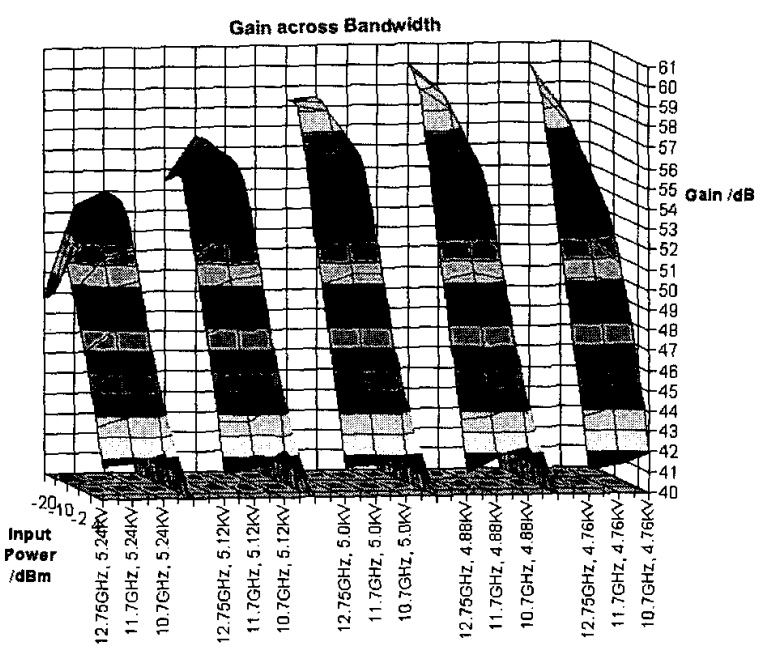

